\title{
Remote Viewing Image Mosaic based on Fuzzy Cellular Automata Corner Detection in Substation
}

\author{
LI Jing \\ Electronic Department, Henan Mechaniacal and Electrical Engineering \\ College,China \\ hnjzlijing@163.com
}

\begin{abstract}
In order to improve the effect of remote video monitoring system, a new image mosaic technology based on fuzzy cellular automata corner detection is presented. Firstly, the edge feature points from two images are extracted by fuzzy cellular automata; secondly the corner feature points are extracted by corner detection method based on geometry feature of corner; thirdly, the corresponding feature point pairs are got by the cross-correlation of the gray scale around the corner feature points; finally the images can be stitched by matched corner feature point pairs. The experiment of remote viewing image mosaic in substation shows that this method can achieve image mosaic effectively and it will be benefit to improve the safety and reliability of substation operation.
\end{abstract}

Keywords: substation remote video monitoring system, image mosaic, fuzzy model cellular automata, corner, edge detection

\section{Introduction}

Current remote video monitoring system in substation can not fully play its due role in real-time active supervision [1]. In order to improve the effect of remote video monitoring system, good mosaic technology must be presented. For a complete surveillance image, we must use image mosaic technology to piece the surveillance images together. Currently image mosaic field of image processing has become an important research aspect.

Image mosaic method involves two main steps: image registration and image fusion. The core technology of image mosaic is image registration technique. The most commonly used image registration techniques mainly include three types: feature-based image registration, gray-based image registration and transform domain-based image registration. Current mainstream image mosaic method is feature-based image registration [2]. The basic image features includes region, texture, contour, edge and corner, etc. Edge and corner are the most basic features of all. But the existing methods are usually carried out using only one of the characteristics of image registration, either edge features or corner features [3-7]. So the number of feature points is very large by the existing methods, and the calculation time is large too. Although edge points and corner points are different characteristics, they are associated too [8]. Usually the corners of the image are the edges of the images, and the corners are the intersections of two or more boundary lines. If we can use the relationship between edge and corner, we can get the feature points which are edge points and corner points simultaneously. So we can improve the image registration accuracy and reduce the computation amount of image registration. In this paper, we adopt the fuzzy cellular automata edge detection method [9] to get the edges of the remote viewing image images in substation, 
and then extract the corner feature points by corner detection method based on geometry feature of corner.

\section{Edge Detection based on Fuzzy Cellular Automata}

There are many edge characteristics information used edge detection of images, everyone has excellences itself. So how to fuse this information should be discussed. In the article [4], a method based on fuzzy cellular automata fusing direction information and edge order information is put forward. This method can get the thin and sequential edges which is the useful to getting corner feature points based on geometry feature of corner

\subsection{Cellular Automata}

Cellular automata (CA) [10] is a dynamical system in which time and space are discrete. The cells are arranged in the form of a regular lattice structure and each must have a finite number of states. These states are updated synchronously according to a specified local rule of interaction. The most elementary composing parts of CA are cells, cell space neighborhoods and rules. The formula is $\mathrm{G}=(S, N, R) \cdot \mathrm{G}$ refers to the state of the system. $\mathrm{S}$ is the state of cells. $\mathrm{N}$ is the relationship between neighbor and $\mathrm{R}$ is the rules of evolvement. The following state of each objective cell is based on the present state and evolvement rules of its neighbor cells.

Nowadays, CA has been used widely in sociology, biology, ecology, information science, computer science, physics, mathematics and many other scientific research fields. But it is still the underway step to put CA into image processing.

\subsection{Direction Information Measure}

Direction information measure [11] can be taken into edge detection of images. It is supposed that the coordinate of current pixel point is $(i, j)$, pixel matrix is $I\left(i_{i, j}\right) . N(i, j)$ refers to the Moore neighborhood. $l_{\theta}$ is a direct line which cross the centre point and has a angle $\theta$. This line divide $\mathrm{N}(\mathrm{i}, \mathrm{j})$ into two parts: $\mathrm{S}_{\theta 1}$ and $\mathrm{S}_{\theta 2}$. So the definition of Direction Information Measure M(i,j) is as following:

$$
M_{i, j}=d_{\theta \max }-d_{\theta \min }
$$

There into:

$$
\begin{gathered}
d_{\theta \max }=\max _{0^{\circ} \leq \theta \leq 180^{\circ}}\left(d_{\theta}\right) \\
d_{\theta \min }=\min _{0^{\circ} \leq \theta \leq 180^{\circ}}\left(d_{\theta}\right) \\
d_{\theta}=\left|f_{S \theta 1}-f_{S \theta 2}\right| \\
f_{S \theta 1}=\sum_{(i, j) \leq S \theta 1} a_{i, j} \\
f_{S \theta 2}=\sum_{(i, j) \in S \theta 2} a_{i, j}
\end{gathered}
$$


At last a direction information measure matrix which takes matrix $\mathrm{M}_{(\mathrm{i}, \mathrm{j})}$ as matrix I can be gotten. When the current point direction information measure is large, it can show that there is an edge which goes across in the neighborhood. Contrarily, there isn't.

\subsection{Edge Order Measure}

The edge of the image not only has the characteristic of gray scale mutation but also shows some order characteristic of its neighborhood. Actually, edge has the neighborhood characteristic, just as follows:

1) There is a gray scale mutation on the edge.

2) Edge is not only a boundary between attributive (gray scale) area.

3) Edge area has a width.

4) Edge has its directivity.

5) Edge has its continuity.

A single point can not be called the edge. So when chose an edge point, not only to consider the directivity, but also need to care the orderliness of its neighbor.

The author of article [12] has offered two parameters about the edge order measure:

1. The neighbor edge intensity is defined as follows:

$$
E_{g}=\frac{\left|g_{1}-g_{2}\right|}{\left|g_{\text {max }}-g_{\text {min }}\right|}
$$

The maximum and minimum gray scale in the whole image are respectively expressed by $g_{\max }, g_{\min }$. The two neighbors' average gray scales are respectively expressed by $g_{1} 、 g_{2}$. A larger neighbor edge intensity $\mathrm{E}_{\mathrm{g}}$ shows a greater full change of the neighbor gray scale, as well as a better orderliness, and all of these may lead to a possibility to be the edge.

2. The width of the neighbor edge isolation is defined as follows:

$$
\begin{gathered}
E_{d}=\frac{E_{d 0}}{n+1} \\
E_{d 0}=\left\|P_{1}-P_{2}\right\|=\sqrt{\left(P_{1 x}-P_{2 x}\right)^{2}+\left(P_{1 y}-P_{2 y}\right)^{2}}
\end{gathered}
$$

Here $\mathrm{P}_{1} \& \mathrm{P}_{2}$ respectively refers to the two geometrical centers in two neighbors. A larger neighbor edge width $\mathrm{E}_{\mathrm{d}}$ shows a greater possibility in different gray scale area, as well as a better orderliness, and all of these may lead to a possibility to be the edge.

In an ideal edge condition, here it takes a $(4+1) \times(4+1)$ rectangle neighbor, so $n=2$. Before measurement, it is necessary to calculate the neighbor edge intensity matrix: $\mathrm{E}_{g(i, j)}$ and the width of the neighbor edge isolation matrix: $\mathrm{E}_{d(i, j)}$.

\subsection{Fuzzy Rule and Fuzzy Measure}

The main purpose of fuzzy inference is to simulate the decision-making of human brain which is based on the fuzzy logic and approximate inference. As a rule, a fuzzy "IF (condition), THEN(conclusion)" will be taken to show the relationship between the input and output of a system. Considering that there is one feature that can affect the 
judgment of the edge and the judgment has its fuzziness, it will take a fuzzy consequence to do a fuzzy measure of the edge which contains one fuzzy input and one fuzzy output.

The input is the information of the direction and the order information (neighbor edge intensity and the width of the neighbor edge isolation). These fuzzy sets are \{large, small\}. And the corresponding input membership function adopts a triangle, just as follows:

1. The membership function of the direction information measure

The membership function which shows "large" is:

$$
\mu_{1}(\mathrm{M})=\left\{\begin{array}{cc}
0 & 0 \leq M \leq T_{1} \\
\left(M-T_{1}\right) /\left(T_{2}-T_{1}\right) & T_{1} \leq M \leq T_{2} \\
1 & T_{2} \leq M \leq 1
\end{array}\right.
$$

The membership function which shows “small” is:

$$
\mu_{2}(\mathrm{M})=\left\{\begin{array}{cc}
1 & 0 \leq M \leq T_{1} \\
1-\left(M-T_{1}\right) /\left(T_{2}-T_{1}\right) & T_{1} \leq M \leq T_{2} \\
0 & T_{2} \leq M \leq 1
\end{array}\right.
$$

Usually $\mathrm{T}_{1}=\mu+\sigma, \mathrm{T}_{2}=\mu+2 \sigma, \mu$ is desired value, $\sigma$ is standard deviation, $\mu=4.7, \sigma=29.8, \mathrm{~T}_{1}=34.5, \mathrm{~T}_{2}=64.3$.

2. The membership function of neighbor edge intensity

The membership function which shows "large" is:

$$
\mu_{1}\left(\mathrm{E}_{\mathrm{d}}\right)=\left\{\begin{array}{cc}
\frac{\mathrm{E}_{d}}{T_{3}} & 0 \leq E_{d} \leq T_{3} \\
1 & T_{3} \leq E_{d} \leq 1
\end{array}\right.
$$

The membership function which shows "small” is:

$$
\mu_{2}\left(E_{d}\right)=\left\{\begin{array}{cc}
1-\frac{E_{d}}{T_{3}} & 0 \leq E_{d} \leq T_{3} \\
0 & T_{3} \leq E_{d} \leq 1
\end{array}\right.
$$

Usually $\mathrm{T}_{3}=1 / 3$.

3. The membership function of the width of the neighbor edge isolation

The membership function which shows "large" is: 


$$
\mu_{1}\left(\mathrm{E}_{\mathrm{g}}\right)=\left\{\begin{array}{cc}
\frac{\mathrm{E}_{g}}{T_{4}} & 0 \leq E_{g} \leq T_{4} \\
1 & T_{4} \leq E_{g} \leq 1
\end{array}\right.
$$

The membership function which shows "small” is:

$$
\mu_{2}\left(\mathrm{E}_{\mathrm{g}}\right)=\left\{\begin{array}{cc}
1-\frac{\mathrm{E}_{g}}{T_{4}} & 0 \leq E_{g} \leq T_{4} \\
0 & T_{4} \leq E_{g} \leq 1
\end{array}\right.
$$

Usually $\mathrm{T}_{4}=\frac{2 \sqrt{2}}{3}$.

The output fuzzy sets are \{yes, no\}, which can show whether the point is an edge point. The rule of fuzzy inference is: if three input is "large", the input is "yes", otherwise it's "no".

\subsection{Anti-fuzzy}

An anti-fuzzy method is used to feed back the matrix $M$ of direction information measure. Its theory is that: to decide the edge point by the fuzzy measurement; For all of the edge points, the Moore neighborhood which takes those as the centre point is taking into consideration, if its neighborhood accords with the edge structure, an edge point in the hypo-neighborhood of the neighborhood edge point may be existed, the hypo-neighbor of the largest direction information measure is to be found among each neighborhood edge point; There is no hurry to take this point as the edge point directly, but to give increment feedback to the direction information measure, at the next moment, another fuzzy measurement of the new direction information measure should be taken to decide the edge point. This increment is got from the anti-fuzziness of the fuzzy measure of the centre cell.

The membership function which shows “yes” is:

$$
\mathrm{Z}=\lambda \mu_{o} \quad 0 \leq \mu_{o} \leq 1
$$

The membership function is a half-triangle. $\mu_{o}$ is the output grade of membership function which shows "yes". The coefficient $\lambda$ has an effect on the width of edge judgment. $\lambda=\sigma / 2=14.9$, is taken in the test which gets a great effect.

\subsection{Rules of fuzzy CA model}

The regulations of CA are as follows:

1. We calculate Direction information measure matrix $M$, neighbor edge intensity matrix $E_{g}$, width of the neighbor edge isolation matrix $E_{d}$ and get edges by fuzzy rule. If the point is edge point, the relevant $\mathrm{b}$ of matrix $\mathrm{B}$ is 1 , or else is 0 . 
2. If $\mathrm{b}_{i, j}=1$. Look at its moore neighborhood. If the number of the edge points in its neighbor is greater than or equal to two, then find out the location of the neighbor cell whose state is 1 and sign the corresponding place on the matrix $M$ of direction information measure. Find out the cell whose direction information measure value is the biggest in its hypo-neighborhood for each founded neighbor cell. Make sure that the following state information measure value is the sum of the present value and the output value of anti-fuzziness.

3. If $b_{i, j}=1$. If the number of the edge points in its neighbor is smaller than two, that is to say, it is not the edge point and its next state will be 0 .

4. CA begins to evolve, until get to a stable state $\left(\mathrm{B}^{t}=\mathrm{B}^{t+1}\right)$.

Finally, we can get the edge of image.

\section{Corner Detection Method based on Geometry Feature of Corner}

Based on the article [3], usually the corners of the image are the edges of the images, and the corners are the intersections of two or more boundary lines. According this corner geometry, firstly we preprocess the two original images; secondly the thin and sequential edge feature points from two images are extracted by fuzzy cellular automata; thirdly define some graphical templates, see in Figure 1, Based on these templates, we can scan image edge points to get corner points.

In Figure 1(a), if the template center is the mid-point of a boundary line, the template center is a edge point instead of a corner point. In Figure 1(b), if the template center is the intersection of two boundary lines, the template center is a corner point. In Figure 1(c), if the template center is the intersection of many boundary lines, the template center is a corner point too. We can get corner points based on graphical templates.

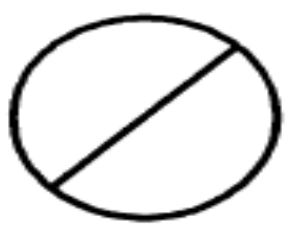

(a)

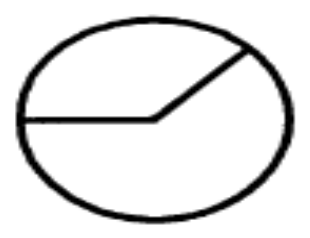

(b)

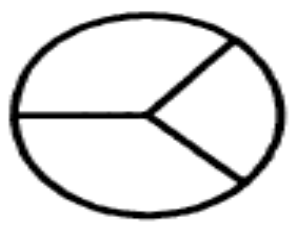

(c)

Figure 1. Graphical Templates

Corner extraction of the basic principle is as follows:

$$
\left\{\begin{array}{c}
X=\sum_{i=1}^{n} X_{i}-X_{0} \\
y=\sum_{i=1}^{n} Y_{i}-Y_{0} \\
Z=X^{2}+Y^{2}
\end{array}\right.
$$


In formula $17, \mathrm{X}_{\mathrm{i}}, \mathrm{Y}_{\mathrm{i}}$ are the $\mathrm{i}$ 'th pixel of the $\mathrm{x}$-coordinate value and y coordinate values in the template inner boundary line. $\mathrm{n}$ is the number of boundary pixels in the template (not contain central point).

In the corner extraction process, we also may not use strictly circular templates. Taking boundary pixel as the center, we get the four pixels in every the boundary line which is connected with the central point. The four pixels are the boundary points which are the nearest of the central point of every boundary line. Of course, we can also take the three pixels in every the boundary line, that is depending on the size of the template. We assign a $\mathrm{Z}$ value to each one corresponding boundary pixel, and every $\mathrm{Z}$ values are initialized to 0 , also define a corner point determination threshold $T$. For Figure 1a, and figure $1 \mathrm{~b}$, we calculate the value of the point $\mathrm{Z}$ based on the above formula. For Figure 1a in the case, the two boundary pixel lines are symmetrical, and the center point is the point of symmetry. According to the formula 17, we can calculate the $\mathrm{X}$ value and $\mathrm{Y}$ value, strictly these values should all be 0 . However, due to the digitization of images, there is an error, the $\mathrm{X}, \mathrm{Y}$ value may be 0 or a small integer, so the $\mathrm{Z}$ value of corresponding points is small. For Figure $1 \mathrm{~b}$ in the case, there is no significant symmetric case, the values of $\mathrm{X}$ and $\mathrm{Y}$ are always large, so the $\mathrm{Z}$ value of corresponding points is large too by formula 17. For Figure 1c in the case, the pixel of the template center is the intersection point of the three or more boundary lines, and the pixel of the template center must be a corner point. So we set the $\mathrm{Z}$ value of the pixel of the template center a larger value than the determination threshold value $T$, in experiment the $Z$ value is typically set to 60 . After getting all $\mathrm{Z}$ values of the boundary pixel, if a $\mathrm{Z}$ value is greater than the threshold $\mathrm{T}$ and is the maximum value of the local area, it is determined that this point is a corner point. This local area may be the $5 \times 5$ or $7 \times 7$ pixel template which uses this point as center. If a boundary pixel $\mathrm{Z}$ value is less than the threshold $\mathrm{T}$ of determination corner, it must not be a corner.

\section{Image Mosaic based on Corner Points}

After extracting the corner features of surveillance images, we will locate the boundaries range for splicing. This is done by pre-calibration of the camera position. The two cameras with the same internal parameters are set in the appropriate position. So the overlapping portion of two images will be known.

After getting the overlapping portion of two images, we will count the maximum similarity $[13,14]$ between two images pixel values in the overlapping portion of two images. We should find every point in image 1 has a corresponding point in image 2 only, and the two points are regarded as the same position.

Let $X_{1}\left(x_{1}, y_{1}\right), X_{2}\left(x_{2}, y_{2}\right)$ respectively be two random characteristic points of the image $\mathrm{I}_{1}$ and image $\mathrm{I}_{2}$, and the correlation coefficient is defined as follows:

$$
\operatorname{Cor}\left(X_{1}, X_{2}\right)=\frac{\operatorname{cov}\left(X_{1}, X_{2}\right)}{\operatorname{std}\left(X_{1}\right) \times \operatorname{std}\left(X_{2}\right)}
$$

In the formula $18, \operatorname{std}(\cdot), \operatorname{cov}(\cdot, \cdot)$ are the standard deviation and correlation function as follows:

$$
\operatorname{std}(X)=\sqrt{\frac{\sum_{i=-n}^{n} \sum_{j=-n}^{n}[I(x+1, y+1)-M(X)]^{2}}{W}}
$$




$$
\operatorname{Cov}\left(X_{1}, X_{2}\right)=\frac{\sum_{i=-n}^{n} \sum_{j=-n}^{n}\left[I_{1}\left(x_{1}+i, y_{1}+j\right)-M\left(X_{1}\right)\right]\left[I_{2}\left(x_{2}+i, y_{2}+j\right)-M\left(X_{2}\right)\right]}{W}
$$

In the formula 20, $\mathrm{M}(\mathrm{X})$ is pixel gray average value of relevant part in image $\mathrm{I}_{1}$ and $\mathrm{I}_{2}$.

$$
M(X)=\frac{\sum_{i=-n}^{n} \sum_{j=-n}^{n} I(x+i, y+j)}{W}
$$

So, in the two images for matching, we select one point $(\mathrm{x}, \mathrm{y})$ as the initial point in image 1 , and roughly locate the corresponding image point ( $\left.x^{\prime}, y^{\prime}\right)$ in another image 2 depending on translation component in the direction of mosaic. Next, we set certain steps $\mathrm{i}, \mathrm{j}$, and according to the principle of maximum cross-correlation coefficient do iterative search until the best splice point is acquired. Finally, the images can be stitched by the best matched corner feature point pairs based on coordinate transformation.

\section{Experiment of Image Mosaic}

In this experiment we chose two surveillance images in one current remote video monitoring system of substation. The two images are related to the same transformer, but the two images can not show the whole picture of the transformer. So if we want to get the whole picture of the transformer, we must stitch the two images.

The size of image 1 is $551 \times 428$, and the size of image 2 is $558 \times 428$, as shown in Figure 2 . Firstly, we get the edge feature points based on fuzzy cellular automata method above, and the results are in Figure 3, we can see the edges are thin and sequential. Secondly, we get the corner feature points based on geometry feature of corners in Figure 4, we can see these points are both edges and corners and the number of these points is small. Therefore, this method can improve the accuracy of image registration and reduce the computation amount of image registration. Finally, we get the results of image mosaic by formula 18, 19, 20, 21, results as shown in Figure 5. The result of image mosaic in this experiment show that this method can achieve image mosaic effectively and it is suitable for image mosaic needs in substation remote video system.

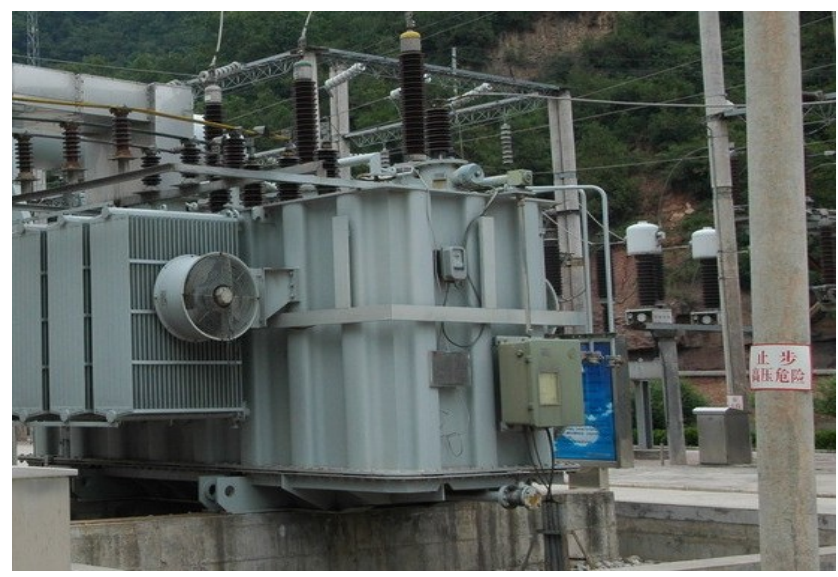

(a) Image 1 


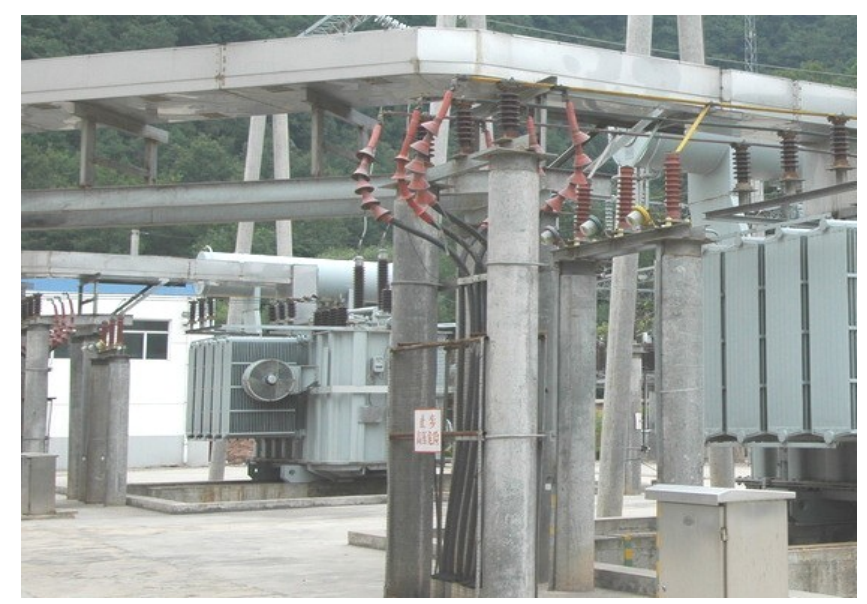

(b) Image 2

Figure 2. Two Surveillance Images

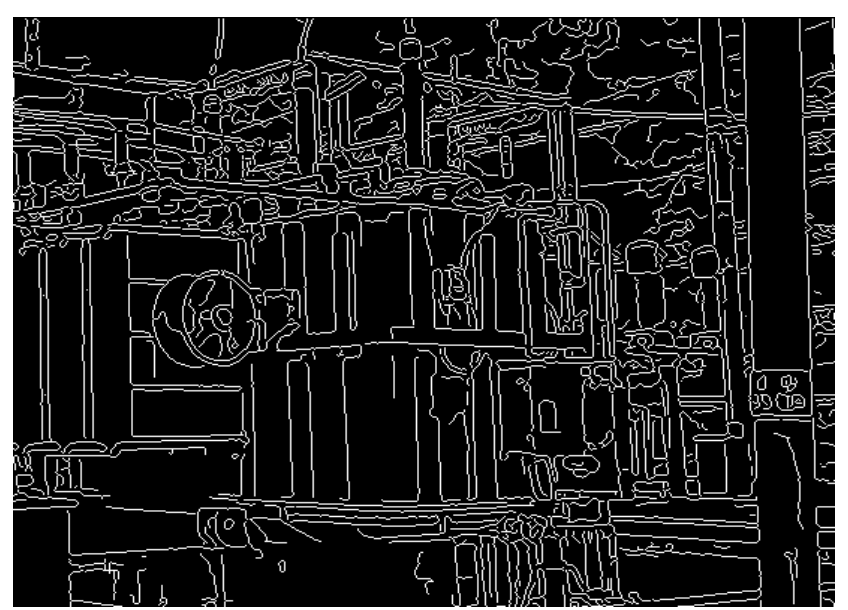

(a) Image 1

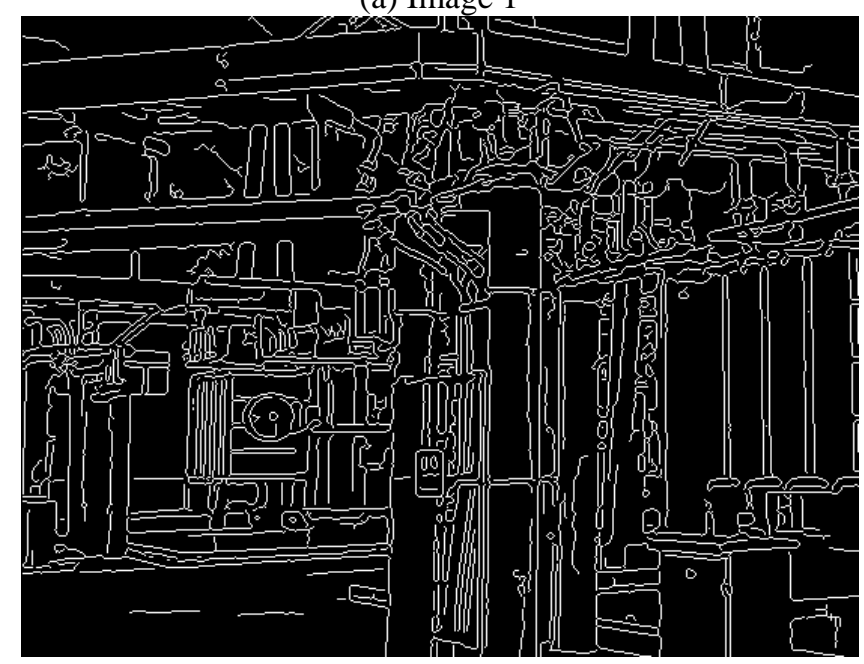

(b) Image 2

Figure 3. Edges of Figure 2 


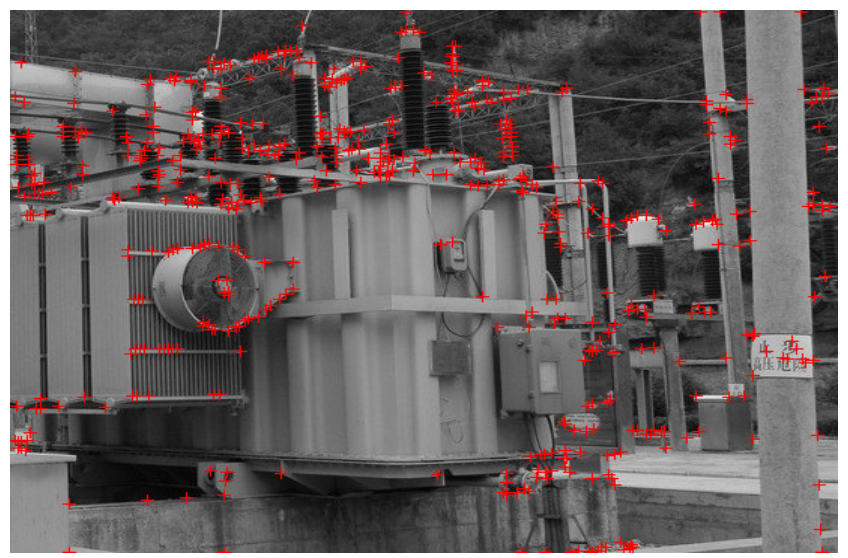

(a) Image 1

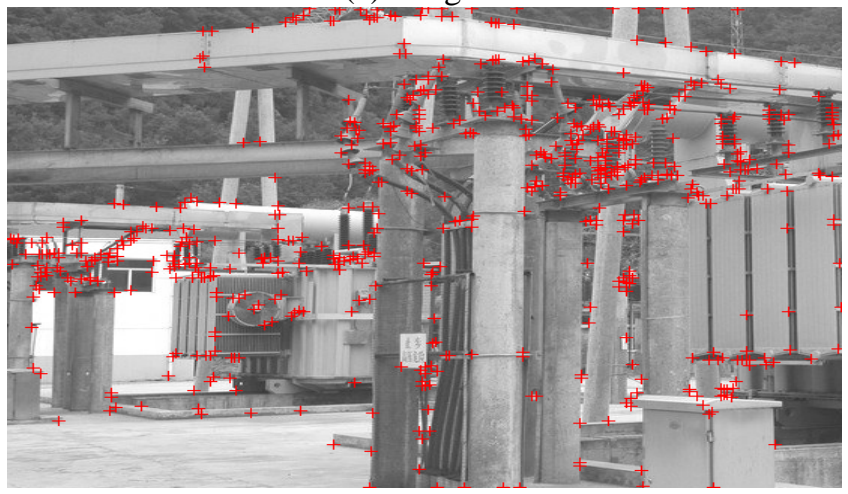

(b) Image 2

Figure 4. Corners of Figure 2

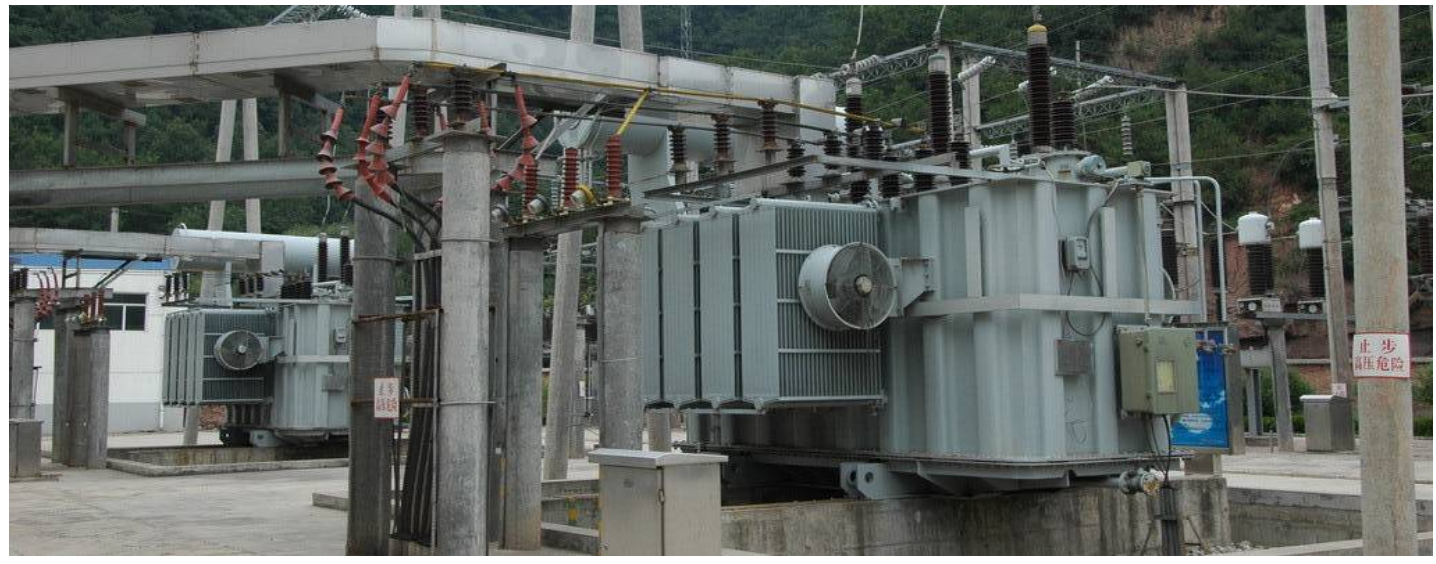

Figure 5. Spliced Image of Figure 2

\section{Conclusion}

The substation remote viewing image mosaic method based on fuzzy cellular automata corner detection is a good attempt to improve the effect of remote video monitoring system in substation. This method has the following advantages:

1) It can get the thin and sequential edges. 
2) Iit can improve the accuracy of image registration and reduce the computation amount of image registration.

3) It can achieve image mosaic effectively and it is suitable for image mosaic needs in substation remote video system.

\section{References}

[1] Y. Y. Sang, S. L. Zheng. Investigation and realization of Tele-Video system for provincial substation. Proceedings of the CSU-EPSA. 1, 21(2009)73-77

[2] X. Chen. Image mosaic algorithm based on feature points auto-matching. Optics \& Optoelectronic Technology. 6, 7 (2009)41-44

[3] J. F. Canny. A computational approach to edge detection, IEEE Trans Pattern Analysis and Machine Intelligence. 6 , 8 (1986)679-698

[4] L. Kitchen, A. Rosenfeld. Gray-level corner detection.Pattern Recognition Letters. 1(1982)95 -102

[5] H. Wang, M. Brady. Realtime corner detection algorithm formotion estimation.Image and Vision Computing. 9, 13 (1995)675-703

[6] C. Harris, M. Stephens. A combined corner and edge detector. International Joint Conference on Artificial Intelligent. (1997)

[7] M. Trajkovic, M. Hedley. Fast corner detection.Image and Vision Computing. 1, 16 (1998)75-87

[8] Q. B. Zou, D. X. Zhou and X. P. Cai. A robust algorithm of image corner detection based on thinning edges. Computer Applications and Software. 23(3) (2006)110-112

[9] K. Zhang, Z. Li, X. O. Zhao. Edge detection of images based on fuzzy cellular automata. Proceedings of the SNPD. (2007)289-294

[10] C. H. Zhou, Z. L. Sun and Y. C. Xie, Research of Geography Cellular Automata, Science Press, Bei Jing (1999)

[11] X. Yang, D.Q. Liang. Multiscale edge detection based on direction information. Journal of Xiadian University. 4, 24 (1997)524-530

[12] X. H. Pei, X. Yang, W. X. Wie. Multiscale edge detection based on fuzzy measure in neighborhood. Journal of China Institute of Communications. 20 (1999)37-41

[13] S. Y. Liu, L. Xia. Fabric image mosaic based on cellular automata. Journal of Textile Research. 1, 32 (2001)29-33

[14] Y. J. Hou, J. Cao. An image mosaic method based on harris corner feature. Informatization Research. 10, 36 (2010)23-25

[15] David O. Aborisade, Novel Fuzzy logic Based Edge Detection Technique, IJAST Volume 29,(2011) April,pp.75-82

[16] Y. Tyagi, T. A. Puntambekar, Preeti Sexena and Sanjay Tanwani, A Hybrid Approach to Edge Detection using Ant Colony Optimization and Fuzzy Logic, IJHIT Vol. 5, No.1, (2012)January ,pp.37-46

[17] Kossi Edoh, Edge Detection with Geometric Transforms and Isotropic Nonlinear Equation, IJSIP Vol. 4, No. 3, (2011)September ,pp.51-60

\section{Author}

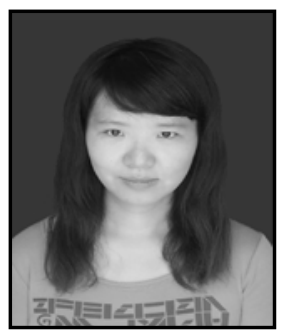

LI Jing, received the B.Eng degree in Electronic and Information Engineering from Henan Normal University and the M.Eng degree in Control Engineering from Wuhan University of Technology ,CHINA in 2003 and 2008 respectively. She is a teacher of Henan Mechanical and Electrical Engineering College,CHINA.Her currently research interests on Power monitoring system and remote automatic control system. 
International Journal of Security and Its Applications Vol.7, No.6 (2013) 